\title{
Analysis of system transient response and its effect on sound speed measurement based on TFCW
}

\author{
Chao Zhou ${ }^{\mathrm{a}}{ }$, Chunjie Qiao ${ }^{\mathrm{b}}$, Zhigang Huang $^{\mathrm{c}}$ and Weihua Dai ${ }^{\mathrm{d}}$ \\ College of Mechatronics Engineering and Automation, National University of Defense Technology \\ Changsha, 410073, China \\ azhouchaowhu@126.com, bqiaoxj@nudt.edu.cn, ch-zhigang@163.com, ${ }^{d} 13755027628 @ 163 . c o m$
}

Keywords: Sound speed, Measurement system, Transient response.

Abstract. This paper carries out a research to analyze system transient response and its effect on sound speed measurement based on two frequency continuous wave (TFCW). Because the bandwidth of an actual measurement system is limited, the received signal is generally polluted by a system transient response, and it can significantly degrade the measurement accuracy of sound speed. We consider the actual system as a bandpass system. The phenomenon of transient response can be clearly noted, and simulations show that its effect on sound speed measurement is related to the starting time, the observation length of signal and the system bandwidth. To validate our research, we finally designed an experimental system to perform a sound speed measurement, and the experimental results are consistent with the simulations.

\section{Introduction}

Sound speed of seawater, as one of important physical quantities, plays a crucial role in ocean-related science research and engineering practice [1-3]. Because of the complexity of the ocean's environment, generally, sound speed is not a constant, but randomly distributes within a range. Many researchers have focused on the accurate sound speed measurement. Estimation of the time-of-flight (TOF) is a novel technique for sound speed measurement [4-5]. The concept is based on a kinematic formula: $C=D / \mathrm{TOF}$, where $C$ is the sound speed to be measured, $D$ is the distance between the transmitter and the receiver. TOF is the time that the ultrasonic signal travels.

Among the methods of TOF estimation, two frequency continuous wave (TFCW) is an excellent approach, which can provide a highly accurate estimate [7-8]. The TOF is extracted by calculating the phase shift between the transmitted and received signals, and the initial step of TFCW is identical to method based on single frequency continuous wave (SFCW). The superiority of TFCW compared to SFCW is the concomitant extension of measurable range of sound speed.

For an actual measurement system, transient response, as a general problem, has an adverse effect on the measurement of sound speed [4]. Because the system bandwidth is limited, the received signal is accompanied by transient response signal that can significantly degrade the performance of TOF estimation, therefore, hamper the sound speed accuracy. This paper aims to study the generation principle of transient response in measurement system, and analyze the effect on the TFCW sound speed measurement. It can provide supports for design and implementation of the measurement system, so as to improve the accuracy loss introduced by system transient response. The rest of paper is organized as follows. In section 2, the measurement of sound speed based on TFCW is discussed. In section 3, we make analysis of the principle of transient response and its effect on sound speed measurement. In section 4, an experiment is demonstrated and conclusions are given in section 5 .

\section{Sound speed measurement based on TFCW}

Basic principle. The basic principle of sound speed measurement based on TOF estimation is shown in Fig. 1. Two transducers are mounted on different sides along the same path, the TOF is the time that the ultrasonic signal takes to travel from the transmitter to the receiver. If $s(t)$ and $x(t)$ are the transmitted and the received signals, respectively, the TOF can be extracted by calculating the phase 
shift between $s(t)$ and $x(t)$. Then, the sound speed is calculated by $C=D / \mathrm{TOF}$, where the distance $D$ is a known variable.

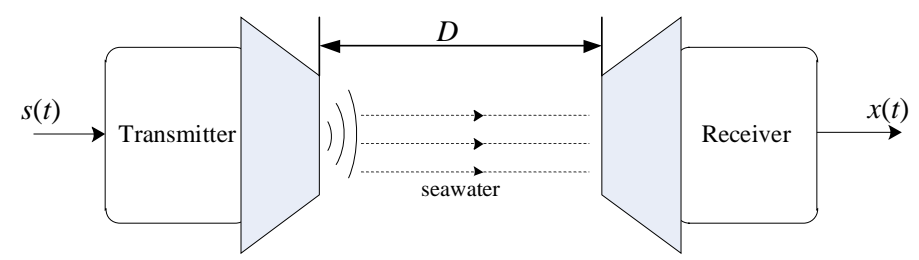

Fig. 1. The basic principle of sound speed measurement based on TOF estimation The transmitted signal $s(t)$ is typically expressed as

$$
s(t)=\cos \left(2 \pi f_{1} t\right)+\cos \left(2 \pi f_{2} t\right),
$$

where $f_{1}$ and $f_{2}$ are the two designed frequencies, and we assume that $f_{1}>f_{2}$. If $s(t)$ propagates in the medium, then the received continuous wave signal can be described as

$$
\left\{\begin{array}{l}
x(t)=\alpha s\left(t-\tau_{0}\right)+n(t) \\
\tau_{0} \in\left[\tau_{\min }, \tau_{\max }\right]
\end{array},\right.
$$

where $\alpha$ is the amplitude of the received signal and $\tau_{0}$ is the actual value of the TOF, which is limited in a given range $\left[\tau_{\min }, \tau_{\max }\right]$. The key to achieve an accurate sound speed measurement is to obtain a TOF result that is close to $\tau_{0}$ within an acceptable accuracy.

The method of TOF estimation utilizing TFCW is as follows. The continuous wave $s(t)$ is transmitted, and simultaneously the received signal $x(t)$ is sampled. By using a discrete Fourier transform technique, the phase shift $\left(\varphi_{1}\right)$ at first frequency $\left(f_{1}\right)$ between the transmitted signal and the received signal is calculated. In the same way, the second phase shift $\left(\varphi_{2}\right)$ at $f_{2}$ would be given and we have $0 \leq \varphi_{1}<2 \pi, 0 \leq \varphi_{2}<2 \pi$. The following will estimate the TOF by comparing the two phase shifts:

$$
\left\{\begin{array}{l}
2 \pi f_{1} \cdot\left(\mathrm{TOF}-\tau_{\text {min }}\right)=2 k_{1} \pi+\phi_{1} \\
2 \pi f_{2} \cdot\left(\mathrm{TOF}-\tau_{\text {min }}\right)=2 k_{2} \pi+\phi_{2}
\end{array},\right.
$$

where

$$
\left\{\begin{array}{l}
\phi_{1}=\bmod \left(\varphi_{1}-2 \pi f_{1} \tau_{\text {min }}, 2 \pi\right) \\
\phi_{2}=\bmod \left(\varphi_{2}-2 \pi f_{2} \tau_{\text {min }}, 2 \pi\right)
\end{array},\right.
$$

and both $\square_{1}$ and $\square_{2}$ are restricted in $[0,2 \pi)$. The integers $k_{1}$ and $k_{2}$ have only two possible values: $k_{1}=$ $k_{2}$ and $k_{1}=k_{2}+1$. From Eq. (3), the TOF result can be expressed as

$$
\mathrm{TOF}=\frac{\Delta \phi}{2 \pi\left(f_{1}-f_{2}\right)}+\tau_{\min },
$$

where the phase difference $\Delta \square$ can be defined by the following algorithm: if $\square_{1}>\square_{2}$, then $\Delta \square=\square_{1}$ $\square_{2}$, else $\Delta \square=\square_{1}+2 \pi-\square_{2}$. Subsequently, the sound speed to be measured would be given by $C=D$ / TOF.

Ideal model of measurement system. The system model of sound speed measurement is shown in Fig. 2 , where $s(t), x(t)$ and $n(t)$ are the transmitted signal based on TFCW, the received signal and the added noise, respectively. $h(t)$ represents the unit impulse response of the measurement system which consists of a transmitting transducer, a receiving transducer, propagation medium and signal conditioners, etc. 


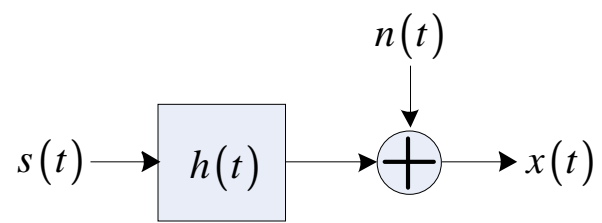

Fig. 2. The system model of sound speed measurement.

Without noise, the received signal $x(t)$ can be expressed as

$$
x(t)=s(t) * h(t) .
$$

From Eq. (2), the mapping relationship we expect between $s(t)$ and $x(t)$ should be an ideal time delay model, and $h(t)$ and its Fourier transform are expected to be

$$
\left\{\begin{array}{l}
h(t)=\alpha \delta\left(t-\tau_{0}\right) \\
H(j \Omega)=\alpha e^{-j \Omega \tau_{0}}
\end{array} .\right.
$$

In this paper, we define the measurement system satisfies Eq. (7) as an ideal measurement system. It has two important features: (1) The bandwidth of ideal measurement system is unlimited and its amplitude gain $\alpha$ is a constant; (2) The phase frequency curve of ideal measurement system is linear and its slope equals to the time of acoustic signal propagation in the medium. The ideal measurement system is just a carrier of sound speed which will not lead to measurement error.

Practically there must be measurement error introduced by the measurement system. Firstly, the bandwidth of the actual system is limited so that the received signal is accompanied by transient response signal that can degrade the performance of TOF estimation. Meanwhile, large error also occurs because of the group delay of actual system. In the following paper, we only analyze the principle of transient response and its effect on sound speed measurement.

\section{The effect of transient response on sound speed measurement}

Analysis of the system transient response. We assume that $s(t)$ is transmitted at time zero, then it can be written as

$$
s(t)=\left[\cos \left(2 \pi f_{1} t\right)+\cos \left(2 \pi f_{2} t\right)\right] u(t)
$$

For linear time invariant (LTI) system, the received signal $x(t)$ can be expressed as

$$
x(t)=s(t) * h(t)=\int_{-\infty}^{\infty} h(\tau) s(t-\tau) d \tau .
$$

Substituting (8) to (9), then we have

$$
\left\{\begin{array}{l}
x(t)=x_{1}(t)+x_{2}(t) \\
x_{1}(t)=\int_{-\infty}^{t} h(\tau) \cos \left(2 \pi f_{1}(t-\tau)\right) d \tau, \\
x_{2}(t)=\int_{-\infty}^{t} h(\tau) \cos \left(2 \pi f_{2}(t-\tau)\right) d \tau
\end{array}\right.
$$

where $x_{1}(t)$ and $x_{2}(t)$ are the received signals at $f_{1}$ and $f_{2}$, respectively, and both $x_{1}(t)$ and $x_{2}(t)$ consist of a steady-state response and a transient response. For example, $x_{1}(t)$ can be decomposed as: $x_{1}(t)=x_{1 s}(t)$ $+x_{1 t}(t)$, where $x_{1 s}(t)$ denotes the steady-state response and $x_{1 t}(t)$ denotes the transient response, and they are given by 


$$
\left\{\begin{array}{l}
x_{1 s}(t)=\int_{-\infty}^{\infty} h(\tau) \cos \left(2 \pi f_{1}(t-\tau)\right) d \tau \\
x_{1 t}(t)=-\int_{t}^{\infty} h(\tau) \cos \left(2 \pi f_{1}(t-\tau)\right) d \tau
\end{array} .\right.
$$

If $h(t)$ represents an measurement system without bandwidth limit, from Eq. (7), we have $x_{t}(t)=x_{1 t}(t)+$ $x_{2 t}(t)=0 t \geq \tau_{0}$ that means the transient response does not exist in the received signal.

Consider the measurement system as a strict bandpass system, the transfer function is described as

$$
H(j \Omega)= \begin{cases}1 & , \Omega_{0}-\frac{B}{2} \leq|\Omega| \leq \Omega_{0}+\frac{B}{2}, \\ 0 & , \text { otherwise }\end{cases}
$$

where $\Omega_{0}$ denotes the center frequency and $B$ is the system bandwidth. By using an inverse Fourier transform, the system impulse response can be given by

$$
\begin{aligned}
h(t) & =\frac{1}{2 \pi} \int_{-\infty}^{\infty} H(j \Omega) e^{j \Omega t} d \Omega \\
& =\frac{1}{\pi} \frac{1}{t}\left\{\sin \left[\left(\Omega_{0}+B / 2\right) t\right]-\sin \left[\left(\Omega_{0}-B / 2\right) t\right]\right\} .
\end{aligned} .
$$

From (12) and (10), the transient response $x_{1 t}(t)$ at $f_{1}$ is deduced, and the system transient response $x_{t}(t)$ can be given subsequently. Fig. 3 shows the transient response curves. The transient response of ideal system is always equal to zero. The transient response of bandpass system is a fluctuating curve and constantly decaying, where the center frequency and bandwidth of measurement system are set at $1 \mathrm{MHz}$ and $400 \mathrm{kHz}$, respectively, and the frequencies of the transmitted signal are set at $f_{1}=975 \mathrm{kHz}$ and $f_{2}=1025 \mathrm{kHz}$. Fig. 4 shows the waveform of the received signals. Because of the additive transient response, the received signal of bandpass system has a waveform distortion compared to the ideal system especially at the initial time.

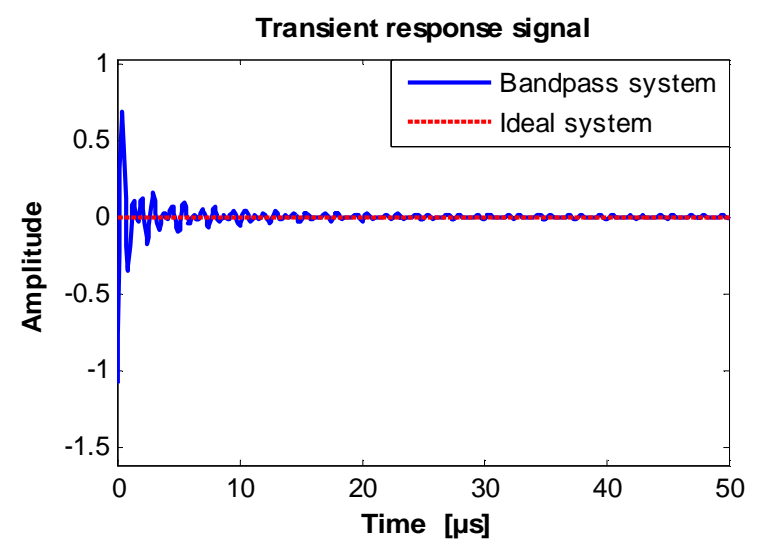

Fig. 3. The system transient response signal.

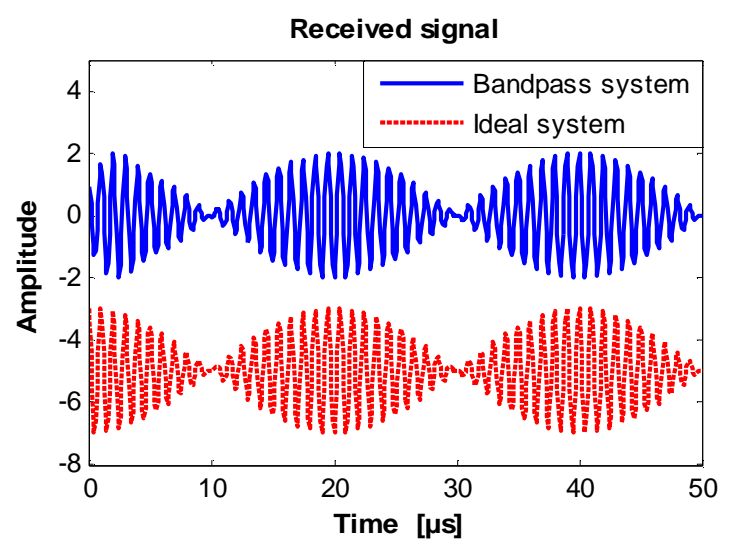

Fig. 4. The received signal.

Effect of the transient response on sound speed measurement. For an actual measurement system, the transient response can significantly degrade the sound speed accuracy, and it is related to the starting time, the observation length of signal and the system bandwidth. Fig. 5 shows the TOF error result from system transient response, where the center frequency and bandwidth of measurement system are set at $1 \mathrm{MHz}$ and $400 \mathrm{kHz}$, respectively, and the frequencies of the transmitted signal are set at $f_{1}=975 \mathrm{kHz}$ and $f_{2}=1025 \mathrm{kHz}$. In Fig. 5(a), as the starting time $\left(t_{p}\right)$ goes on, TOF error is fluctuant in the local, but slowly reduces in the whole. The TOF error achieves the maximal value $5.0 \times 10^{-3} \mu \mathrm{s}$ when $t_{p}=4 \mu \mathrm{s}$, and if $t_{p} \geq 20 \mu \mathrm{s}$, the TOF error is less than $4.4 \times 10^{-4} \mu \mathrm{s}$. In Fig. 5(b), TOF error curves in different signal observation length are demonstrated, and it is shown that the longer the signal, the smaller the TOF error. However, the observation length can not be increased without a limit due to the 
received signal is also accompanied by echoes. Fig. 5(c) shows the TOF error result from transient response in different system bandwidth. Obviously, the wider the bandwidth, the smaller the TOF error. Table 1 proves these conclusions in detail.

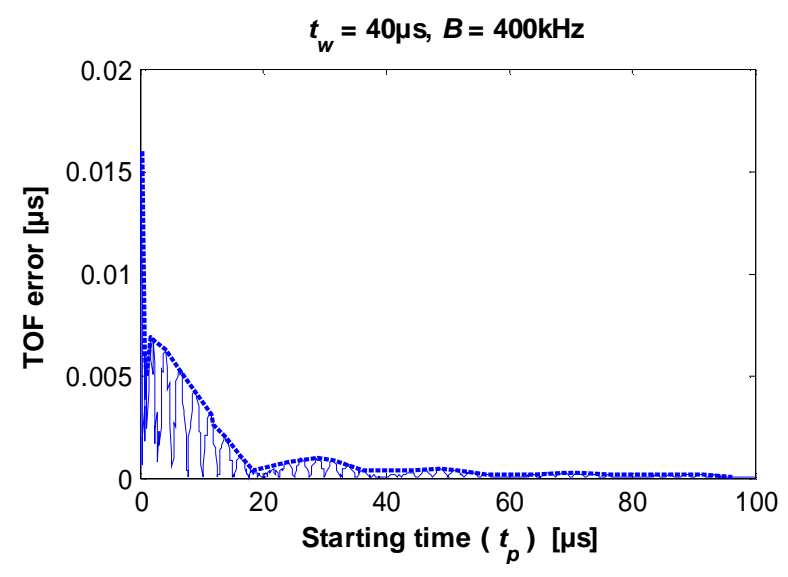

(a)

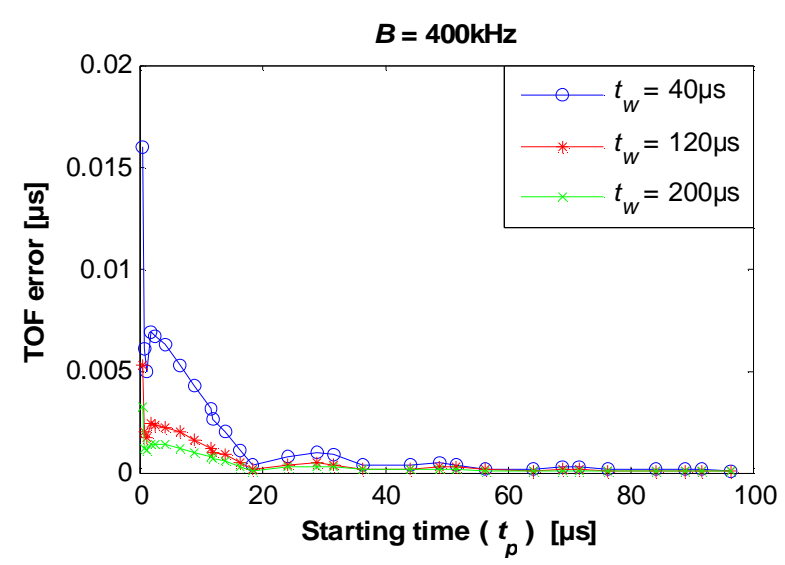

(b)

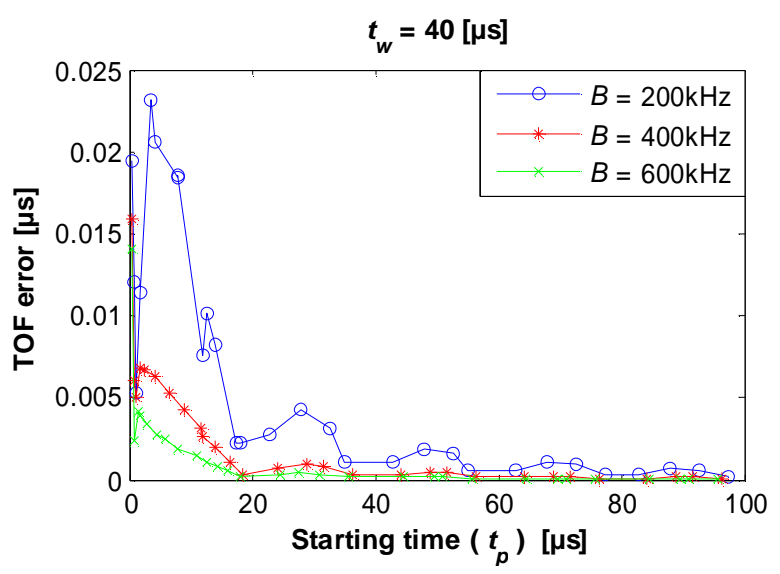

(c)

Fig. 5. The TOF error results from transient response: (a) The TOF error curve as starting time goes on. (b) The TOF error in different observation length. (c) The TOF error in different system bandwidth.

Table 1. The effect of transient resopnse on TOF estimation and sound speed measurement

\begin{tabular}{c|ccc}
\hline Maximal TOF error & $200 \mathrm{kHz}$ & $400 \mathrm{kHz}$ & $600 \mathrm{kHz}$ \\
\hline Starting time & & & \\
\hline \multirow{2}{*}{$t_{p} \geq 0 \mu \mathrm{s}$} & $2.3 \times 10^{-2} \mu \mathrm{s}$ & $1.6 \times 10^{-2} \mu \mathrm{s}$ & $1.2 \times 10^{-2} \mu \mathrm{s}$ \\
& $\left(1.7 \times 10^{-4}\right)$ & $\left(1.2 \times 10^{-5}\right)$ & $\left(9.0 \times 10^{-5}\right)$ \\
\hline \multirow{2}{*}{$t_{p} \geq 20 \mu \mathrm{s}$} & $4.2 \times 10^{-3} \mu \mathrm{s}$ & $9.5 \times 10^{-4} \mu \mathrm{s}$ & $4.2 \times 10^{-4} \mu \mathrm{s}$ \\
& $\left(3.2 \times 10^{-5}\right)$ & $\left(7.1 \times 10^{-6}\right)$ & $\left(3.2 \times 10^{-6}\right)$ \\
\hline
\end{tabular}

Note: The value in '( )' represents the relative accuracy of sound speed while the distance and the signal observation length are set at $D=0.2 \mathrm{~m}, t_{w}=40 \mu \mathrm{s}$.

\section{Experiments}

Experimental setup. To study the transient response of an actual measurement system and analyze its effect on sound speed measurement, we designed an experimental system and performed a sound speed measurement in distilled water. The experimental setup is shown in Fig. 6 and essentially consists of two ultrasonic transducers, a signal transceiver module, a PC, a thermostatic water bath, a frequency meter, and a trough. 


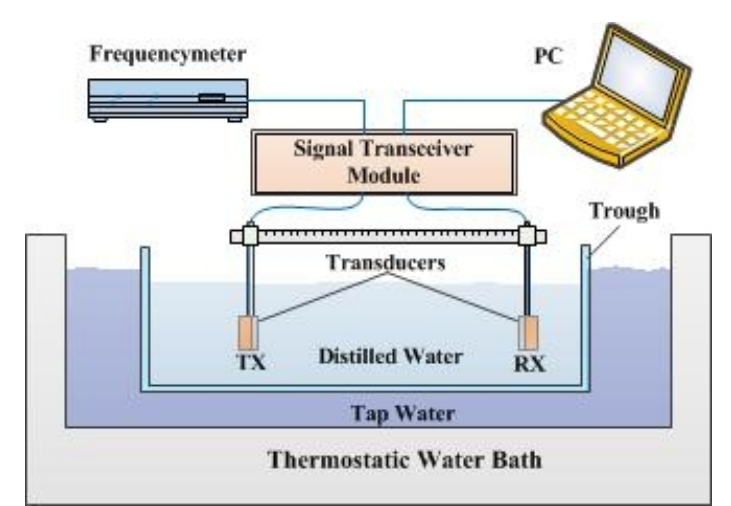

(a)

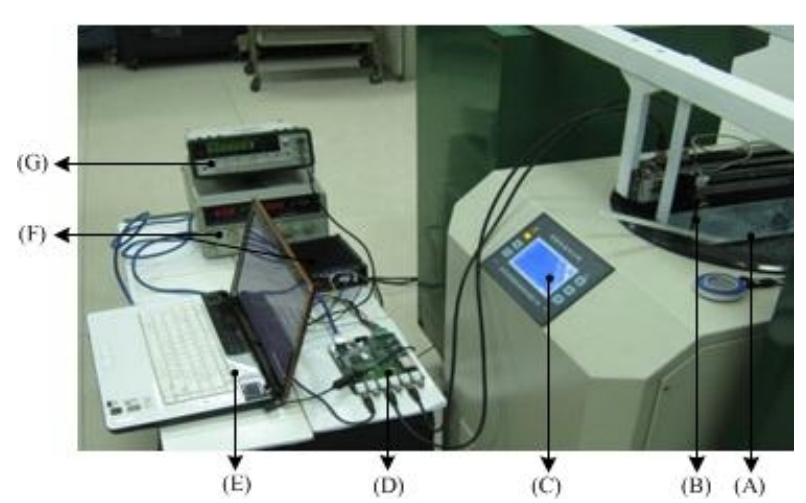

(b)

Fig. 6. The experimental setup: (a) Schematic of the experimental system. (b) Picture of the actual experimental system. (A) is the trough with distilled water, (B) is one of the transducers, $(\mathrm{C})$ is the thermostatic water bath, (D) is the signal transceiver module, (E) is the PC, (F) is the power supply and $(\mathrm{G})$ is the frequency meter.

The two transducers, operated at $1 \mathrm{MHz}$, are fixed relative to each other and fully immersed in the distilled water. The distance $D$ between them can be determined by the rule on the slide guide. The transmitted signal is generated on the PC, downloaded through the Ethernet to the signal transceiver module. When the PC sends a trigger command or an external trigger occurs, the DAC works synchronously with the ADC sampled at $10 \mathrm{msps}$, and the signal sampled is uploaded to the PC for processing to output final results. The entire system works on $50-\mathrm{MHz}$ clock. The thermostatic water bath is to construct a stable experimental environment.

Experimental results. Based on extensive testing work, the bandwidth of the experimental system is about $400 \mathrm{kHz}$, and the frequencies of transmitted signal are set at $f_{1}=975 \mathrm{kHz}, f_{2}=1025 \mathrm{kHz}$, respectively. Fig. 7(a) shows the waveform of the received signal. We can clearly see the phenomenon of waveform distortion, especially at the initial time. By removing the steady-state response in the received signal, we pick up the transient response signal polluted by noise, which is shown in Fig. 7(b).

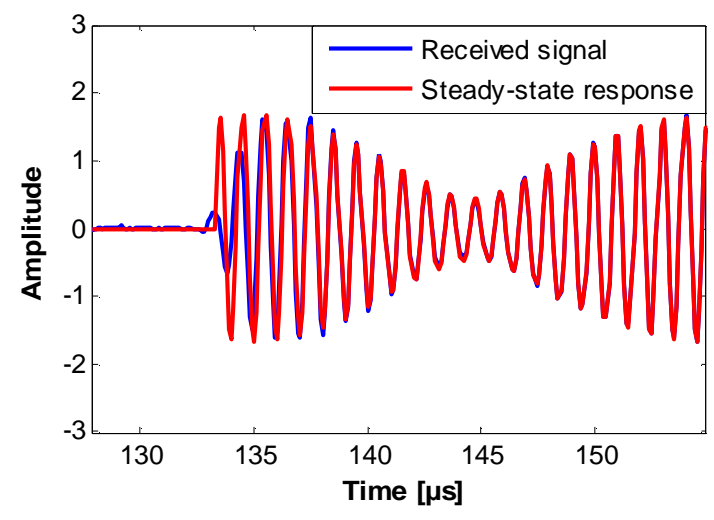

(a)

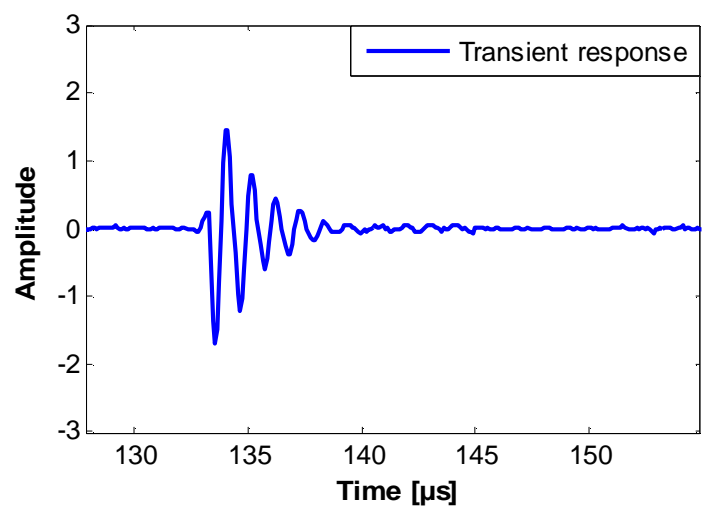

(b)

Fig. 7. The signal waveform in our experiment: (a) The received signal and the reconstructed steady-state response. (b) The transient response polluted by noise.

By using the method of TOF estimation in section 2, an TOF result can be deduced and the sound speed to be measured can be given by $C=D / \mathrm{TOF}$, where the distance $D=0.185 \mathrm{~m}$. In order to suppress the effect of noise, we performed 40 times experiments, and Figure 8(a) shows the results of TOF estimation where the yellow curve is the average result. The TOF result with the transient response is close to zero is regarded as the actual value, then the TOF error is shown in Fig. 8(b). The trend of TOF error in our experiment is consistent with Fig. 5, which validates the analysis method for transient response and its effect on sound speed measurement. 


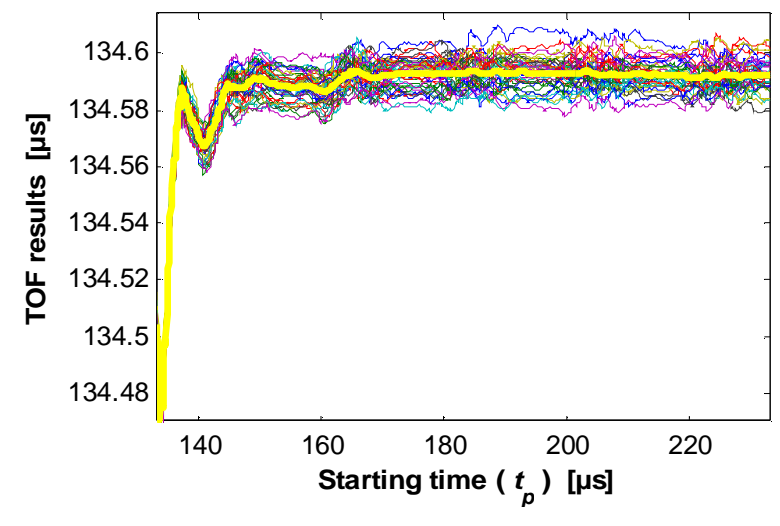

(a)

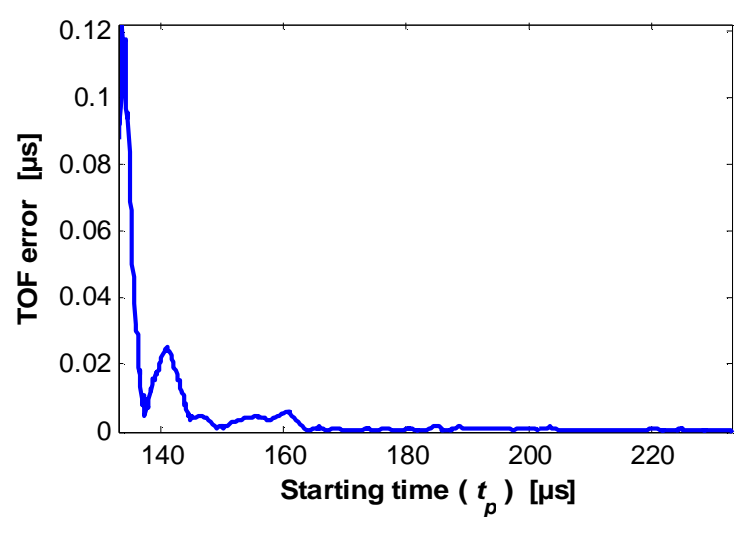

(b)

Fig. 8. The results of TOF estimation: (a) The results of 40 times experiments. (b) The TOF error.

\section{Conclusions}

For an actual system of sound speed measurement, the received signal is generally polluted by a transient response due to the limited system bandwidth, and it can significantly degrade the sound speed accuracy. In this paper, we carry out a research to analyze the system transient response and its effect on sound speed measurement based on TFCW. The simulations show that the effect of transient response on sound speed measurement is related to the starting time, the observation length of signal and the bandwidth of measurement system. It can provide supports for the design and implementation of a sound speed measurement system in practice. We also set up an experimental system and the experimental result validates our research work.

\section{References}

[1] J.P.M Trusler, Physical Acoustics and Metrology of Fluids., Adam Hilger, 1991.

[2] A. D. Sweeney, C. D. Chadwell and J. A. Hildebrand, Calibration of a Seawater Sound Velocimeter, IEEE Journal of Oceanic Engineering, 31(2), (2006), 454-461.

[3] D. D. Brian, F. W Peter and D. C. Bruce, On equations for the speed of sound in seawater, The Journal of the Acoustical Socitety of America, 93(1), (1993), 255-275.

[4] C. J. Qiao, W. H. Dai and C. Zhou, A novel method of sound speed measurement based on Parameter-estimation of continuous wave, Proceedings of IEEE 11th International Conference on Electronics Measurement and Instruments, (2013), 110-115.

[5] G. Eaton and D. T. Dakin, Miniature Time of Flight Sound Velocimeter Offers Increased Accuracy over Sing-Around Technology and CTD Instrumentation, Proceedings of the Oceanology International '96 Conference, 1996.

[6] D. Marioli, C. Narduzzi, C. Offelli, D. Petri, E. Sardini and A. Taroni, Digital Time-of-Flight Measurement for Ultrasonic Sensors, IEEE Trans. on Instrum. Meas., 41(5), (1992), 93-97.

[7] K. N. Huang and Y. P. Huang, Multiple-frequency ultrasonic distance measurement using direct digital frequency synthesizers, Sens. Actuators A, 149(1), (2009), 42-50.

[8] W. Y. Tsai, H. C. Chen, and T. L. Liao, High accuracy ultrasonic air temperature measurement using multi-frequency continuous wave, Sens. Actuators A, 132(2), (2006), 526-532. 